\title{
Terminology Value Set
}

National Cancer Institute

\section{Source}

National Cancer Institute. Terminology Value Set. NCI Thesaurus. Code C113497.

Terms drawn from one or more terminologies and defined as a separate set, generally

for specific coding purposes and given a formal designation. 